\title{
Dermatologist and Patient Perceptions of Treatment Success in Alopecia Areata and Evaluation of Clinical Outcome Assessments in Japan
}

\author{
Jake Macey $\cdot$ Helen Kitchen · Natalie V. J. Aldhouse $\cdot$ Russel T. Burge • \\ Emily Edson-Heredia (D) · Jill S. McCollam • Yoshitaka Isaka • \\ Hitoe Torisu-Itakura
}

Received: October 26, 2020 / Accepted: December 12, 2020 / Published online: January 10, 2021

(C) The Author(s) 2021

\begin{abstract}
Introduction: The content validity and treatment success thresholds of clinical outcome assessments (COAs) for alopecia areata (AA) including the Alopecia Areata-Investigator Global Assessment ${ }^{\mathrm{TM}}$ (AA-IGA $\left.{ }^{\mathrm{TM}}\right)$, Scalp Hair Assessment Patient-Reported Outcome ${ }^{\mathrm{TM}}$ (PRO), and clinician-reported outcome (ClinRO) and PRO measures for eyebrows, eyelashes, eye irritation, and nails-were established in interviews with dermatologists and patients in North America. This study aimed to confirm the content validity and treatment success thresholds of these measures with clinicians and patients in Japan.
\end{abstract}

Supplementary Information The online version contains supplementary material available at https:// doi.org/10.1007/s13555-020-00477-6.

J. Macey · H. Kitchen · N. V. J. Aldhouse

Clinical Outcomes Assessment, DRG Abacus (Part of Clarivate), Manchester, UK

R. T. Burge · E. Edson-Heredia $(\bowtie) \cdot J$. S. McCollam Eli Lilly and Company, Indianapolis, IN, USA e-mail: eheredia@lilly.com

R. T. Burge

Winkle College of Pharmacy, University of

Cincinnati, Cincinnati, OH, USA

Y. Isaka $\cdot H$. Torisu-Itakura

Medical Development Unit, Eli Lilly Japan K.K.,

Kobe, Japan
Methods: Qualitative interviews were conducted in Japan with dermatologists with AA expertise and adults with AA who experienced $\geq 50 \%$ scalp hair loss. Interviews included concept elicitation and cognitive interview questions. Data were analyzed using thematic and framework techniques.

Results: Seven dermatologists and 15 patients participated. Scalp hair loss was the most important sign/symptom of AA and the greatest treatment priority. Dermatologists and patients understood the AA-IGA ${ }^{\mathrm{TM}}$, Scalp Hair Assessment $\mathrm{PRO}^{\mathrm{TM}}$, and other COAs, and found these measures to be appropriate, relevant, and clinically meaningful. Dermatologists and patients confirmed that achieving $\leq 20 \%$ scalp hair loss (AA-IGA $^{\mathrm{TM}} /$ Scalp Hair Assessment PRO ${ }^{\mathrm{TM}}$ categories 0 or 1 ) indicated treatment success for patients with $\geq 50 \%$ scalp hair loss. Categories 0 or 1 on the other COAs represented treatment success.

Conclusion: This study confirmed the content validity and treatment success thresholds of the AA-IGA ${ }^{\mathrm{TM}}$, Scalp Hair Assessment $\mathrm{PRO}^{\mathrm{TM}}$, and other ClinRO and PRO measures for AA in Japan. These findings were aligned with interview results in North America and support the use of these measures in AA treatment studies. 


\section{PLAIN LANGUAGE SUMMARY}

About $2 \%$ of people in the world have alopecia areata, which causes them to lose hair on their scalp, face, and body. We interviewed 15 Japanese adults who had lost at least half of the hair on their scalp and seven dermatologists who treated alopecia areata. The dermatologists felt that scalp hair loss was more important to treat than eyebrow and eyelash hair loss. Patients were most bothered about losing their scalp hair and reported feeling anxious or worried about what other people might think about it. Patients and dermatologists were also shown several questionnaires and thought the questionnaires were appropriate to measure the most important symptoms of alopecia areata. Patients considered that a treatment worked well if it gave them at least $80 \%$ of their scalp hair; dermatologists also wanted the treatment to give patients at least $80 \%$ scalp hair. These interviews agree with what has previously been found in interviews with patients and dermatologists in North America.

Keywords: Alopecia areata; Clinical outcome assessment; Clinician-reported outcome; Dermatology; Investigator global assessment; Japan; Patient-reported outcome; Qualitative interview; Treatment success

\section{Key Summary Points}

Why carry out this study?

Alopecia areata is a chronic autoimmune disease that causes hair loss, which can impair patients' quality of life and social functioning.

Information from interviews with dermatologists and patients in North America established the content validity and treatment success thresholds of clinical outcome assessments for alopecia areata, including the Alopecia Areata-Investigator Global Assessment ${ }^{\mathrm{TM}}$, Scalp Hair Assessment Patient-Reported Outcome ${ }^{\mathrm{TM}}$, and clinician-reported and patientreported outcome measures for eyebrows, eyelashes, eye irritation, and nails.
This study aimed to confirm the content validity and treatment success thresholds of these measures with dermatologists and with patients with $\geq 50 \%$ scalp hair loss in Japan.

\section{What was learned from this study?}

Dermatologists and patients understood the measures and confirmed that successful treatment would be hair regrowth resulting in at least $80 \%$ scalp hair.

These findings were aligned with interview results in North America and support the use of these clinically meaningful outcome assessments in alopecia areata treatment studies involving Japanese patients.

\section{DIGITAL FEATURES}

This article is published with digital features, including a summary slide and plain language summary, to facilitate understanding of the article. To view digital features for this article go to https://doi.org/10.6084/m9.figshare. 13365569.

\section{INTRODUCTION}

Alopecia areata (AA) is a chronic inflammatory autoimmune disease characterized by unpredictable, usually patchy, hair loss that can affect the scalp, face, and body [1]. The global prevalence of AA is estimated to be $2 \%$ [2], with a slightly higher prevalence of $2.5 \%$ reported in Japan [3]. AA can substantially impact healthrelated quality of life [4-9]. Most patients with AA experience anxiety, depression, and low selfesteem, which can impair social functioning $[5,6]$. This emotional distress often causes patients to conceal the effects of hair loss by wearing wigs or using cosmetic products $[6,8]$.

No therapy has been approved for AA in the United States (US) [10]. Although some therapies are approved for AA in Japan (e.g., topical 
and intralesional corticosteroids), there is no approved drug with a grade A recommendation (level I or II evidence of effectiveness) according to the Japanese Dermatological Association treatment guidelines [11]. Therapies for AA include anthralin, biologics, calcineurin inhibitors, corticosteroids, minoxidil, prostaglandin analogs, sensitizers, and alternative therapies (e.g., aromatherapy) [1, 12]. Several ongoing large-scale clinical trials are exploring the safety and efficacy of Janus kinase inhibitors for the treatment of severe or very severe AA [13-16]. In small uncontrolled trials, Janus kinase inhibitors were shown to promote hair regrowth in patients with moderate-to-severe AA [17-22].

Content-valid, well-defined, and clinically meaningful outcome measures are required to evaluate therapeutic interventions in global AA clinical trials. The Food and Drug Administration (FDA) recommends incorporating patient perspectives when developing clinical trial endpoints [23-25].

Nine clinical outcome assessments (COAs) for use in clinical trials involving patients with $\geq 50 \%$ AA-related scalp hair loss were developed in line with the FDA Patient-Reported Outcome (PRO) Guidance for Industry [23]. The content validity of these COAs was established in interviews with clinicians and patients in North America.

The Alopecia Areata-Investigator Global Assessment $^{\mathrm{TM}}$ (AA-IGA $^{\mathrm{TM}}$ ) [26] and Scalp Hair Assessment $\mathrm{PRO}^{\mathrm{TM}}$ [27] (Fig. 1) both comprise five severity categories, each associated with a percentage range of scalp hair loss. Clinicians are advised to use the Severity of Alopecia Tool (SALT) [28] to assess the extent of scalp hair loss and apply the SALT score to derive the AA$\mathrm{IGA}^{\mathrm{TM}}$ gradation [26]. In studies conducted in North America, a proposed clinically meaningful response was defined as hair regrowth following treatment resulting in $\leq 20 \%$ scalp hair loss (categories 0 or 1 on the AA-IGA ${ }^{\mathrm{TM}}$ and Scalp Hair Assessment PRO $\left.^{\mathrm{TM}}\right)$ [26, 27].

Clinician-reported outcome (ClinRO) and PRO measures [29] (see Fig. S1 in the "Supplementary Information") were also developed to assess key secondary AA signs/symptoms [30], including the ClinRO Measure for Eyebrow Hair Loss $^{\mathrm{TM}}$, PRO Measure for Eyebrows ${ }^{\mathrm{TM}}$, ClinRO
Measure for Eyelash Hair Loss ${ }^{\mathrm{TM}}$, PRO Measures for Eyelashes ${ }^{\mathrm{TM}}$, ClinRO Measure for Nail Appearance $^{\mathrm{TM}}$, PRO Measure for Nail Appearance $^{\mathrm{TM}}$, and PRO Measure for Eye Irritation ${ }^{\mathrm{TM}}$.

This study aimed to confirm the content validity and treatment success thresholds of the AA-IGA ${ }^{\mathrm{TM}}$, Scalp Hair Assessment PRO ${ }^{\mathrm{TM}}$, and ClinRO and PRO measures for AA-related eyebrow, eyelash, eye irritation, and nail signs/ symptoms through qualitative interviews with dermatologists and patients in Japan.

\section{METHODS}

\section{Clinician and Patient Interviews}

Qualitative, semi-structured clinician and patient interviews were conducted by two experienced Japanese interviewers using concept elicitation and cognitive interviewing techniques. The interviewers received in-person training on the study methodology and semistructured interview guides. The clinician interviews were conducted from August to September 2018, and the patient interviews were conducted from January to February 2019. Clinicians and patients participated separately in $60-$ or $90-\mathrm{min}$ telephone/online or face-toface interviews. Hardcopies of the COAs translated into Japanese were reviewed by both clinicians and patients.

Open-ended concept elicitation questions explored clinicians' and patients' perspectives on AA signs/symptoms (e.g., scalp and other hair loss), the associated impact on daily life (e.g., relationships, functioning, and emotional wellbeing), and the amount (percentage) and quality (e.g., color and thickness) of scalp hair that would be considered a clinically meaningful treatment response. Cognitive interview questions assessed the understanding and appropriateness of the COAs (e.g., clarity of instructions, meaning of terms, and ease of use of response options) [31]. The think-aloud methodology and specific probes explored patients' acceptance and interpretation of these measures [32]. Clinicians were asked to identify the response categories that would represent treatment success for each ClinRO measure. 
a Alopecia Areata-Investigator Global Assessment ${ }^{\mathrm{TM} a}$

\begin{tabular}{|c|c|c|c|c|c|}
\hline & None & Limited & Moderate & Severe & Very Severe \\
\hline $\begin{array}{l}\text { Please rate the patient's scalp } \\
\text { hair loss, as it looks today. }\end{array}$ & $0 \%$ & $1-20 \%$ & $21-49 \%$ & $50-94 \%$ & $95-100 \%$ \\
\hline & $\begin{array}{c}\text { なし } \\
0\end{array}$ & $\begin{array}{c}\text { 限定 } \\
\text { 的 } \\
1 \\
1\end{array}$ & 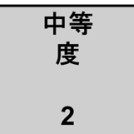 & $\begin{array}{c}\text { 重度 } \\
3\end{array}$ & $\begin{array}{c}\text { 非常に重度 } \\
4\end{array}$ \\
\hline $\begin{array}{l}\text { 現時点での患者の頭皮脱毛の } \\
\text { 程度を評価してください。 }\end{array}$ & $0 \%$ & $1 \sim 20 \%$ & $21 \sim 49 \%$ & $50 \sim 94 \%$ & $95 \sim 100 \%$ \\
\hline
\end{tabular}

(C) Eli Lilly and Company. All rights reserved.

\section{b Scalp Hair Assessment Patient-Reported Outcome ${ }^{\mathrm{TM}}$}
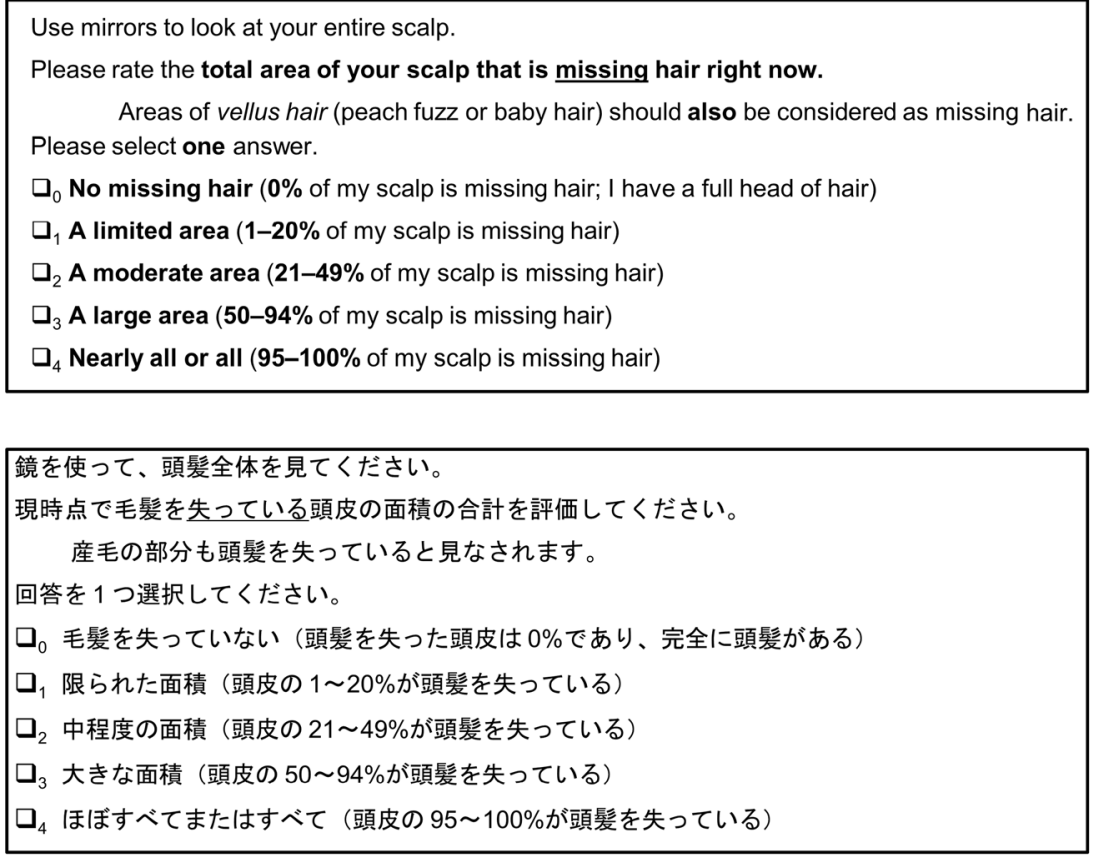

(c) Eli Lilly and Company. All rights reserved.

Fig. 1 The Alopecia Areata-Investigator Global Assessment $^{\mathrm{TM}}\left(\mathrm{AA}-\mathrm{IGA}^{\mathrm{TM}}\right)$ and Scalp Hair Assessment PatientReported Outcome $(\mathrm{PRO})^{\mathrm{TM}}$, with rating instructions provided in English and Japanese. The AA-IGA ${ }^{\mathrm{TM}}$ and Scalp Hair Assessment PRO ${ }^{\mathrm{TM}}$ are subject to copyright owned by Eli Lilly and Company. Permission to use is

Patients were asked to indicate which response category would signify a meaningful treatment response for PRO and ClinRO measures. granted under a Creative Commons Attribution-No Derivatives 4.0 International License. Contact Eli Lilly

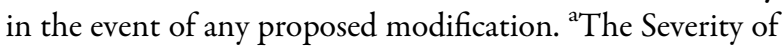
Alopecia Tool [28] is recommended to assess the extent $(0-100 \%)$ of scalp hair loss

\section{Sampling Strategy}

All clinicians and patients were recruited by Breeze, an agency based in Japan, and by Global 
Perspectives, an international agency. Participating clinicians from two sites, a private dermatology clinic and a private university hospital in Tokyo, referred patients to the recruitment agency.

Clinicians were required to have $\geq 5$ years of experience in treating or managing patients with $\mathrm{AA}$, and to treat or manage $\geq 5$ patients with AA (including $\geq 3$ patients with $\geq 50 \%$ scalp hair loss) per month.

Eligible patients were referred by clinicians and provided written informed consent. Male and female patients aged 18-60 years were required to have a diagnosis of severe AA ( $\geq 50 \%$ scalp hair loss lasting more than 6 months with no spontaneous regrowth over the past 6 months), could not have experienced $\geq 95 \%$ scalp hair loss lasting $\geq 8$ years, and could not have other dermatological conditions (e.g., psoriasis and atopic dermatitis) or other forms of alopecia (e.g., androgenetic and telogen) that in the opinion of the clinician may be severe enough to impact the results of this study. Purposive sampling targeted the enrollment of patients with eyebrow and/or eyelash hair loss in addition to scalp hair loss to capture patient experiences of these signs/ symptoms. A target sample size of 15 patients was considered to be adequate for evaluating the content validity of the COAs in Japan $[31,32]$.

\section{Compliance with Ethics Guidelines}

Study procedures and materials aligned with best practice ethical principles for research involving human participants. The patient interview study protocol was approved by the Western Institutional Review Board in the US (ref: 20182441), which provided the single required ethical review of the study per Japanese guidelines.

\section{Analysis}

Audio recordings of the interviews were first transcribed verbatim in Japanese. Transcripts were then translated into English by professional linguists and coded and analyzed using qualitative analysis software (ATLAS.ti, version 7.5) by experienced analysts. Separate codebooks for the clinician and patient interviews were created and used based on the respective interview guides. New codes were created to capture emerging themes and applied to previously coded transcripts. Thematic analysis techniques [33, 34] were applied to concept elicitation data, and framework analysis techniques [35] were applied to cognitive interview data. Identifying information was removed from transcripts and unique identification numbers (IDs) were allocated to anonymize reporting. The ID designated the participant number for clinicians and patients, as well as sex and clinician-reported percentage of missing scalp hair for patients. Quantitative data (e.g., demographics and treatment success thresholds) were analyzed descriptively using Microsoft Excel.

\section{Results}

\section{Clinician Interview Results}

\section{Sample}

Seven dermatologists with AA expertise participated in the interviews (Table 1), and most $(n=6)$ were board-certified by the Japanese Dermatological Association. Clinicians had 8-39 years of experience in regularly treating or managing patients with AA (median 20 years).

\section{Concept Elicitation: AA Signs/Symptoms}

All clinicians observed patients with varying degrees/patterns of patchy scalp hair loss, including complete scalp hair loss, during their practice. They also described patients presenting with eye irritation $(n=5)$, eyebrow and eyelash hair loss $(n=4)$, body hair loss $(n=4)$, nail damage $(n=1)$, and scalp itch $(n=1)$. Scalp hair regrowth was considered the number one treatment priority ( $n=5 / 5$ asked), followed by eyebrow and eyelash regrowth.

\section{Concept Elicitation: Perceptions of Clinically Meaningful Scalp Hair Treatment Success} Quantity of scalp hair $(n=7)$ was the most important factor for determining treatment 
Table 1 Clinician and patient characteristics

\begin{tabular}{|c|c|}
\hline Clinician background and experience & $n=7$ \\
\hline Years treating and/or managing patients with AA, median (range) & $20.0(8.0-39.0)$ \\
\hline \multicolumn{2}{|c|}{ Number of patients treated or managed in a typical month ${ }^{\mathrm{a}}$, median (range) } \\
\hline AA overall & $90.0(30.0-350.0)$ \\
\hline Severe AA ( $50-94 \%$ hair loss) & $45.0(20.0-200.0)$ \\
\hline Very severe AA ( $\geq 95 \%$ hair loss) & $15.0(3.0-50.0)$ \\
\hline Patient demographic and clinical characteristics & $n=15$ \\
\hline Age (years), mean (range) & $36.3(18.0-60.0)$ \\
\hline Male, $n(\%)$ & $9(60)$ \\
\hline \multicolumn{2}{|l|}{ Education, highest level completed, $n(\%)$} \\
\hline High school, currently enrolled & $1(7)$ \\
\hline High school, graduated & $2(13)$ \\
\hline Junior/technical college, graduated & $2(13)$ \\
\hline University graduate school, graduated & $10(67)$ \\
\hline Years since diagnosis, mean (range) & $6.2(1.1-22.1)$ \\
\hline Months of current episode, mean (range) & $3.7(0.8-10.0)$ \\
\hline Clinician-reported percentage of scalp hair loss, mean (range) & $82.5(50.0-100.0)$ \\
\hline Patients with $\geq 95 \%$ scalp hair loss, $n(\%)$ & $9(60)$ \\
\hline Years with $\geq 95 \%$ scalp hair loss, mean (range) & $4.5(0.8-7.0)$ \\
\hline \multicolumn{2}{|l|}{ Treatment received for AA, $n(\%)^{\mathrm{b}}$} \\
\hline Corticosteroids (intralesional/topical/oral) ${ }^{\mathrm{c}}$ & $12(80)$ \\
\hline Topical immunotherapy ${ }^{\mathrm{d}}$ & $4(27)$ \\
\hline Local injection & $2(13)$ \\
\hline Oral antihistamine & $1(7)$ \\
\hline None & $3(20)$ \\
\hline \multicolumn{2}{|l|}{ Concomitant illnesses, $n$ (\%) } \\
\hline Allergic rhinitis & $7(47)$ \\
\hline Atopic dermatitis & $7(47)$ \\
\hline Asthma & $6(40)$ \\
\hline Anxiety & $3(20)$ \\
\hline Depression & $2(13)$ \\
\hline Hypertension & $1(7)$ \\
\hline
\end{tabular}


Table 1 continued

\begin{tabular}{lc}
\hline Patient demographic and clinical characteristics & $n=15$ \\
\hline Psoriasis & $1(7)$ \\
\hline$A A$ alopecia areata \\
a The median value of ranges provided by one clinician (e.g., 40-50) was used to calculate the sample median \\
b Categories were not mutually exclusive \\
c Five patients received eyebrow corticosteroid injections only and three patients received two corticosteroids (topical and \\
intralesional, topical and eyebrow injection, oral and topical) \\
d Topical immunotherapy includes diphencyprone, squaric acid dibutylester, and unspecified
\end{tabular}

success, although hair length $(n=6)$, location of remaining hair loss $(n=5)$, and growth of terminal rather than vellus hair $(n=4)$ were other influential factors. Most clinicians $(n=6)$ reported that hair color did not define treatment success.

Clinicians defined treatment success for patients with $\geq 50 \%$ scalp hair loss at baseline as reaching $60 \%(n=1)$ or $80 \%(n=6)$ scalp hair coverage (i.e., $40 \%$ to $20 \%$ loss). Clinicians ( $n=6 / 6$ asked) reported that attaining $80 \%$ scalp hair after 9 months of treatment was a reasonable timeframe.

\section{Cognitive Interview: $A A-I G A^{T M}$}

All clinicians understood the AA-IGA ${ }^{\mathrm{TM}}$ and considered it an appropriate, relevant measure for assessing scalp hair loss severity (Fig. 1a). Several clarifications to the wording were suggested (e.g., use "Minimal" instead of "Limited"). Five clinicians suggested adding an instruction to exclude vellus hair from the assessment of scalp hair quantity.

Four clinicians considered the AA-IGA ${ }^{\mathrm{TM}}$ response options to represent appropriate gradations of scalp hair loss severity. Two clinicians preferred the cutoff percentages of the AA$\mathrm{IGA}^{\mathrm{TM}}$ to the Japanese clinical guidelines [11] (which divide scalp hair loss into categories at $25 \%$ intervals), as they considered the AA$\mathrm{IGA}^{\mathrm{TM}}$ gradations to be more clinically meaningful. Three clinicians proposed modifications based on their clinical perspective (e.g., combine "Moderate" and "Severe," start "Very severe" at $80 \%$ instead of 95\%) (Fig. 2). None of the suggested changes were critical to understanding the AA-IGA ${ }^{\mathrm{TM}}$.

\section{Treatment Success}

Clinicians reported treatment success as achieving " 1 -limited (1-20\%)" ( $n=5 / 6$ asked) or "2-moderate (21-49\%)" ( $n=1 / 6$ asked) scalp hair loss on the AA-IGA ${ }^{\mathrm{TM}}$ following a 9-month treatment trial (Table 2).

\section{Cognitive Interview: Eyebrow, Eyelash, and Nail ClinRO Measures}

All clinicians understood the ClinRO Measures for Eyebrow Hair Loss ${ }^{\mathrm{TM}}$, Eyelash Hair Loss ${ }^{\mathrm{TM}}$, and Nail Appearance ${ }^{\mathrm{TM}}$ (see Fig. S1a-c in the "Supplementary Information"), and agreed that the response options reflected distinct levels of symptom severity. Noncritical changes to the wording of response options were suggested (e.g., remove "distribution" in the eyebrow measure), but they did not affect clinicians' understanding of the measures.

\section{Treatment Success}

Clinicians considered treatment success as category 0 or 1 on each measure (Table 2).

For the ClinRO Measure for Eyebrow Hair Loss $^{\mathrm{TM}}$, most clinicians ( $n=4 / 5$ asked) considered treatment to be successful if a patient could be categorized as at least " $1-$ minimal gaps in eyebrow hair and distribution is even" at the end of a clinical trial.

For the ClinRO Measure for Eyelash Hair Loss $^{\mathrm{TM}}$, clinicians $(n=3 / 5$ asked) considered treatment to be successful if a patient could be categorized as at least " $1-$ minimal gaps and the eyelashes are evenly spaced along the eyelids on both eyes."

Clinicians' opinions were split for the ClinRO Measure for Nail Appearance ${ }^{\mathrm{TM}}$. Half 


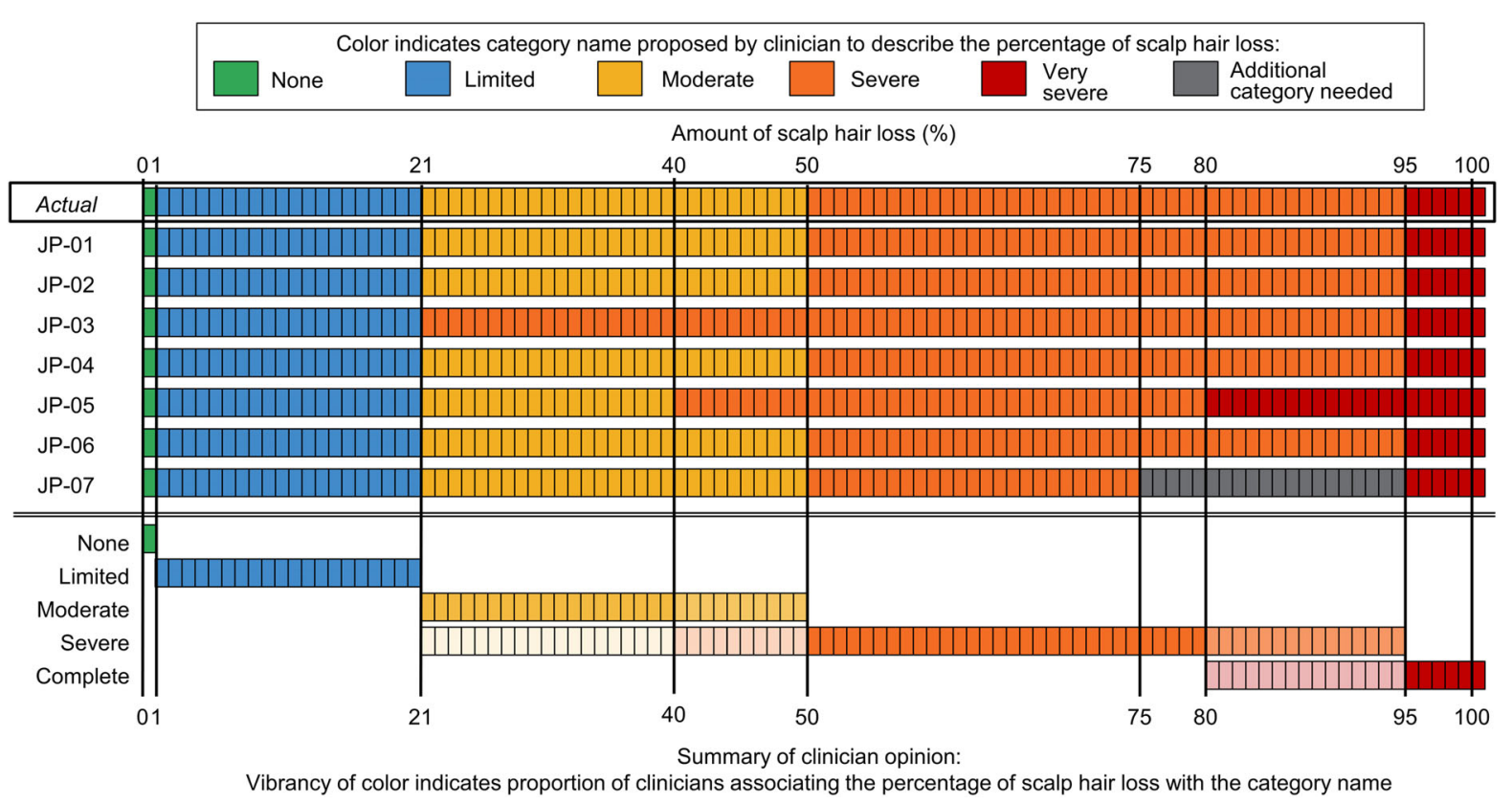

Fig. 2 A color-coded representation of category names proposed by clinicians to describe the percentage of scalp hair loss in the Alopecia Areata-Investigator Global Assessment ${ }^{\mathrm{TM}}$

indicated that treatment would be successful if patients were categorized as " 0 -nails are not at all damaged" ( $n=3 / 6$ asked) or as " 1 -at least one nail is a little damaged" ( $n=3 / 6$ asked). Two of these clinicians did not assess severity of nail damage in clinical practice.

\section{Patient Interview Results}

\section{Sample}

Fifteen patients [60\% male, mean age 36.3 years (range 18-60 years)] participated in the interviews (Table 1). The mean clinician-reported percentage of scalp hair loss was $83 \%$ (range $50-100 \%$ ), and nine (60\%) patients had $\geq 95 \%$ scalp hair loss (mean years with $\geq 95 \%$ scalp hair loss 4.5 years, range 1-7 years). Most patients $(n=10,67 \%)$ experienced scalp hair loss with complete or partial eyebrow and/or eyelash hair loss.

\section{Concept Elicitation: AA Signs/Symptoms and Impact of $A A$ on Daily Life}

Thirteen patients named scalp hair loss as their most bothersome sign/symptom of AA, (e.g., "because that is the area that can be seen" [02-F-
100]). Two patients considered eyebrow hair loss $(n=1)$ or eye irritation $(n=1)$ to be their most bothersome sign/symptom. Patients reported eyebrow hair loss $(n=11)$, eyelash hair loss $(n=8)$, nail damage $(n=2)$, eye irritation $(n=1)$, facial hair loss $(n=1)$, and body hair loss $(n=1)$ among their top 3 most bothersome signs/symptoms (Table S1 in the "Supplementary Information").

Patients described complex and interconnected impacts of AA, which were mostly related to areas of hair loss visible to others. All patients reported how scalp hair loss affected their psychosocial wellbeing, citing anxiety/worry/stress about their AA becoming exposed or how it would progress $(n=12)$, worry about social judgement $(n=11)$, and having feelings of insecurity/inadequacy/selfconsciousness $(n=7)$ and/or sadness $(n=7)$ (Table S2 in the "Supplementary Information"). Common physical impacts of AA included eye irritation $(n=8)$ due to eyebrow/eyelash hair loss and feeling the cold easily $(n=3)$ because of scalp hair loss. 
Table 2 Clinicians' and patients' definitions of treatment success for clinician-reported and patient-reported outcome measures for alopecia areata in Japan

\section{Clinician definition of treatment success ${ }^{a}$}

$A A-I G A^{T M}$

$0-$ None (no missing hair)

1 -Limited (1-20\% missing hair) $(n=5 / 6)$

2-Moderate (21-49\% missing hair) $(n=1 / 6)$

Scalp Hair Assessment PRO ${ }^{T M}$

$$
-
$$

ClinRO Measure for Eyebrow Hair Loss ${ }^{T M}$

0 -Full coverage and no areas of hair loss $(n=1 / 5)$

1 -Minimal gaps in eyebrow hair and distribution is even $(n=4 / 5)$

PRO Measure for Eyebrows ${ }^{T M}$

$$
-
$$

ClinRO Measure for Eyelash Hair Loss ${ }^{T M}$

0 -Eyelashes form a continuous line $(n=2 / 5)$

$1-$ Minimal gaps and the eyelashes are evenly spaced $(n=3 / 5)$

PRO Measures for Eyelashes ${ }^{T M}$

$$
-
$$

\section{ClinRO Measure for Nail Appearance ${ }^{T M}$}

$0-$ Nails are not at all damaged $(n=3 / 6)^{\mathrm{b}}$

1 -At least one nail is a little damaged $(n=3 / 6)^{\mathrm{b}}$

\section{Patient definition of treatment success ${ }^{\mathrm{c}}$}

0-None (no missing hair)

1 - Limited (1-20\% missing hair) $(n=15 / 15)$

0-No missing hair (0\% missing hair; I have a full head of hair)

$1-$ A limited area (1-20\% missing hair) $(n=11 / 15)$

$2-\mathrm{A}$ moderate area (21-49\% missing hair) $(n=4 / 15)$

0 - Full coverage and no areas of hair loss $(n=3 / 15)$

1 -Minimal gaps in eyebrow hair and distribution is even $(n=12 / 15)$

0 -Full eyebrows on each eye $(n=3 / 9)$

1 -Minimal gap(s) or a minimal amount of thinning $(n=6 /$ 9)

0 -Eyelashes form a continuous line $(n=4 / 15)$

$1-$ Minimal gaps and the eyelashes are evenly spaced $(n=9 /$ 15)

2-Significant gaps or the eyelashes are not evenly spaced $(n=2 / 15)$

0 -Full eyelashes on each eyelid $(n=2 / 9)$

1 -Minimal gap or minimal gaps along the eyelids $(n=6 / 9)$

2-Large gap or large gaps along the eyelids $(n=1 / 9)$

$0-$ Nails are not at all damaged $(n=4 / 15)$

$1-$ At least one nail is a little damaged $(n=9 / 15)$

$2-$ At least one nail is moderately damaged $(n=2 / 15)$ 
Table 2 continued

\begin{tabular}{ll}
\hline Clinician definition of treatment success $^{\mathbf{a}}$ & Patient definition of treatment success $^{\mathbf{c}}$ \\
\hline PRO Measure for Nail Appearance & \\
- & $0-$ Nails are not at all damaged \\
& $1-$ At least one nail is a little damaged $(n=6 / 6)$
\end{tabular}

PRO Measure for Eye Irritation ${ }^{T M}$

0 - My eyes have not been irritated $(n=2 / 4)$

$1-$ My eyes have been a little irritated $(n=2 / 4)$

The AA-IGA ${ }^{\mathrm{TM}} /$ Scalp Hair Assessment $\mathrm{PRO}^{\mathrm{TM}}$ comprises five severity categories: 0 -none/no scalp hair missing $(0 \%$ missing hair) to 4-very severe/nearly all or all scalp hair missing (95-100\% missing hair). ClinRO and PRO eyebrow, eyelash, eye irritation, and nail measures comprise four severity categories: 0-normal presentation of eyebrows/eyelashes/nails or no eye irritation to 3-no or barely any eyebrows/eyelashes, very damaged nails, or severe eye irritation. Clinicians and patients were asked to define treatment success considering 9 months of treatment

$A A-I G A^{T M}$ Alopecia Areata-Investigator Global Assessment, ClinRO clinician-reported outcome, PRO patient-reported outcome

${ }^{a}$ Clinicians were only asked about their definition of treatment success of the ClinRO measures, and not all clinicians were asked $(n=5-6)$ because of interview time constraints. For the AA-IGA ${ }^{\mathrm{TM}}$, clinicians defined clinically meaningful change from category 3-severe (50-94\% missing hair) on the AA-IGA ${ }^{\mathrm{TM}}$. For the ClinRO Measure for Eyebrow Hair Loss ${ }^{\mathrm{TM}}$, Eyelash Hair Loss ${ }^{\mathrm{TM}}$ and Nail Appearance ${ }^{\mathrm{TM}}$, clinicians were asked which category would indicate treatment success

b Two of the six clinicians did not assess severity of nail damage in clinical practice

${ }^{c}$ For the PRO measures, patients reported meaningful change from their current category. Only patients who rated themselves as having at least " 1 " on the PRO Measures for Eyebrows ${ }^{\mathrm{TM}}$, Eyelashes ${ }^{\mathrm{TM}}$, Eye Irritation ${ }^{\mathrm{TM}}$, and/or Nail Appearance $^{\mathrm{TM}}$, indicating some level of AA involvement in these locations, were asked about treatment success. For the ClinRO measures, patients were asked which category they would need to be rated as by their doctor

\section{Concept Elicitation: Perceptions of Clinically Meaningful Scalp Hair Treatment Success}

Quantity of scalp hair was the most important factor for determining treatment success. Almost all patients $(n=11)$ defined treatment success as $50 \%$ to $80 \%$ scalp hair (i.e., $50 \%$ to $20 \%$ loss $)$; other patients desired $90 \%(n=3)$ or $95 \%(n=1)$ scalp hair (i.e., $10 \%$ or $5 \%$ loss, respectively). Some patients required hair growth in particular scalp locations $(n=4 / 7$ asked) and sufficient hair density $(n=2)$ for treatment to be considered successful.

\section{Cognitive Interview: Scalp Hair Assessment PRO $^{\text {TM }}$}

All patients understood the Scalp Hair Assessment $\mathrm{PRO}^{\mathrm{TM}}$ (Fig. 1b) and were able to self-report their amount of scalp hair loss, although some noncritical clarifications were suggested (e.g., simplify the instruction "Please rate the total area of your scalp that is missing hair right now").

Most patients $(n=11)$ felt that the percentage ranges in the response categories were appropriate (Fig. 3). The majority $(n=10)$ used only the percentage ranges to choose their response because they found them easier to understand or a better representation of hair loss than the descriptors (e.g., Moderate or Large). Other patients used both the percentages and descriptors $(n=4)$ or just the descriptors $(n=1)$ to select their response. The percentages helped two patients to overcome difficulties in reading kanji characters or understanding the response category descriptors.

\section{Treatment Success}

Patients who rated themselves as " 4 -nearly all/ all 95-100\%" $(n=8)$ reported that reaching "2moderate $21-49 \%$ " $(n=4)$ or " 1 -limited 


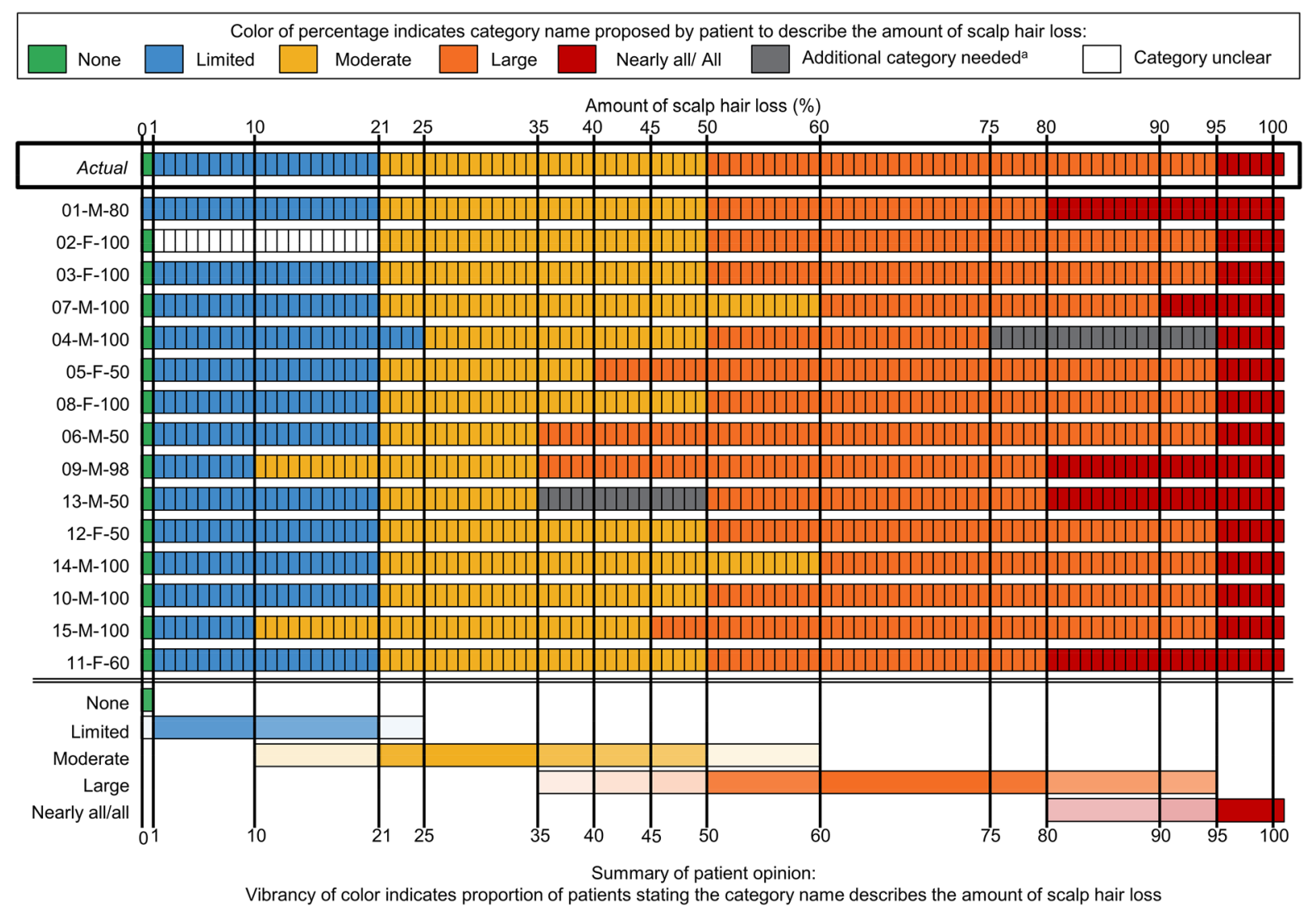

Fig. 3 A color-coded representation of patients' opinions on the percentage ranges included in the Scalp Hair Assessment Patient-Reported Outcome ${ }^{\mathrm{TM}}$ response options. Patient interviews were conducted in the order shown (i.e., from patient 01-M-80 to 11-F-60). ${ }^{a}$ Additional category needed: one patient felt that the category of

1-20\%" $(n=4)$ scalp hair loss after 9 months of treatment could be considered successful. Patients who rated themselves as "3-large 50-94\%" $(n=2)$ or "2-moderate 21-49\%" $(n=4)$ stated that reaching "1-limited 1-20\%" scalp hair loss would indicate successful treatment. One patient reported that remaining in the same "1-limited 1-20\%" scalp hair loss category would represent treatment success.

\section{Cognitive Interview: Eyebrow, Eyelash, Eye Irritation, and Nail PRO Measures}

Most patients understood the PRO Measures for Eyebrows $^{\mathrm{TM}}$, Eyelashes ${ }^{\mathrm{TM}}$, Eye Irritation ${ }^{\mathrm{TM}}$, and Nail Appearance ${ }^{\mathrm{TM}}$ (Fig. S1d-g in the
"Large" was too broad and suggested an additional category between "Large" and "Nearly all/all" for patients with 75-94\% hair loss. One patient felt that there should be an additional category between "Moderate" and "Large" for patients with $35-50 \%$ hair loss

"Supplementary Information") and agreed that the response options corresponded to increasing, distinct levels of symptom severity. Noncritical changes to the wording of response options (e.g., remove "gaps" and "thinning" in the eyebrow measure instructions) were suggested, but these did not prevent patients from providing a response. Eight patients had difficulty differentiating between the response options for the eye irritation measure (e.g., ambiguity of "moderate irritation"). However, six patients provided accurate descriptions of the severity levels, and all $(n=15)$ could provide a response. 


\section{Treatment Success}

Patients supported defining treatment success as category 0 or 1 on each measure (Table 2 ).

Achieving at least category 1 after treatment was considered acceptable by most patients with some level (i.e., self-reported category $\geq 1$ ) of AA-related eyebrow $(n=6 / 9)$, eyelash $(n=6 /$ $9)$, and nail involvement $(n=6 / 6)$ on the respective PRO measures. Patients with eye irritation were split between whether they considered achieving category 0 or $1(n=2 / 4$ each) on the eye irritation PRO measure to be acceptable.

\section{Cognitive Interview: AA-IGA $A^{T M} / C l i n R O$ Measures Treatment Success}

Most patients defined treatment success as achieving at least category 1 on the AA-IGA ${ }^{\mathrm{TM}}$ $(n=15 / 15), \quad$ eyebrow $\quad(n=12 / 15)$, eyelash $(n=9 / 15)$, and nail $(n=9 / 15)$ ClinRO measures (Table 2).

\section{DISCUSSION}

The results of this study support the content validity of the nine COAs for use in AA clinical trials in Japan. Qualitative data collected from interviews with Japanese dermatologists and patients indicated that all nine AA measures were conceptually relevant, appropriate, and clinically meaningful assessments of key AA signs/symptoms. AA was found to substantially impact the health-related quality of life of Japanese patients [4-9].

Interview results were aligned with those in North America [26, 27, 29]. All clinicians and nearly all patients confirmed that scalp hair loss was the most important AA sign/symptom and scalp hair regrowth was the greatest treatment priority. All clinicians $(n=7 / 7 ; 100 \%)$ and most patients $(n=11 / 15 ; 73 \%)$ defined treatment success as reaching $50 \%$ to $80 \%$ scalp hair (i.e., 50-20\% hair loss) during concept elicitation. During cognitive interviews, all clinicians $(n=6 / 6 ; 100 \%)$ and most patients $(n=11 / 15$; $73 \%)$ felt that achieving category " 1 -limited (1-20\%)" scalp hair loss on the AA-IGA ${ }^{\mathrm{TM}} / \mathrm{Scalp}$ Hair Assessment $\mathrm{PRO}^{\mathrm{TM}}$ following a 9-month treatment trial would indicate treatment success. Patient expectations for hair regrowth following treatment varied based on their current hair loss; patients with greater loss reported treatment success even with moderate hair loss remaining. Therefore, categories 0 ( $0 \%$ loss) and 1 (1-20\% loss) were considered the success thresholds for these measures.

Additionally, results confirmed the treatment success thresholds of the ClinRO Measure for Eyebrow Hair Loss ${ }^{\mathrm{TM}}$, ClinRO Measure for Eyelash Hair Loss ${ }^{\mathrm{TM}}$, ClinRO Measure for Nail Appearance $^{\mathrm{TM}}$, PRO Measure for Eyebrows ${ }^{\mathrm{TM}}$, PRO Measure for Eyelashes ${ }^{\mathrm{TM}}$, PRO Measure for Eye Irritation ${ }^{\mathrm{TM}}$, and PRO Measure for Nail Appearance $^{\mathrm{TM}}$. Most clinicians and patients reported that reaching category 1 represented treatment success, and considered category 0 to represent greater improvement. Thus, categories 0 or 1 were considered the treatment success thresholds for each measure.

Several clinicians and patients proposed modifications to the COAs. For example, five clinicians suggested adding an instruction to the AA-IGA ${ }^{\mathrm{TM}}$ to exclude vellus hair from the assessment of scalp hair quantity. However, this was not deemed necessary, as the recommendation is to use the SALT [28] (which requires assessing terminal hair only) to derive the AA$\mathrm{IGA}^{\mathrm{TM}}$ category. No proposed changes were critical to participants' understanding or use of the measures to rate corresponding symptoms.

Our study had several limitations. The English-speaking researchers who developed the COA measures in North America also created the Japanese interview guides and analyzed the interviews but did not conduct the interviews because of language differences. To mitigate inconsistency in study conduct, detailed inperson training sessions on the study methods and interview guide were provided to the native language interviewers, and both teams provided updates/feedback following each interview. In addition, patients with $\geq 50 \%$ scalp hair loss were based in the Tokyo area, and thus the findings may not be generalizable to populations in rural/less urban locations. However, clinicians were from geographically diverse regions in Japan and, therefore, the perspectives of other communities may have been captured. Despite the small sample size of clinicians 
interviewed $(n=7)$, all were highly skilled dermatologists with extensive experience in treating and managing patients with AA. The results of our study provide meaningful insights into clinicians' and patients' perspectives and treatment expectations for AA in Japan.

\section{CONCLUSIONS}

This study confirmed that the content validity and treatment success thresholds were categories 0 or 1 of the AA-IGA ${ }^{\mathrm{TM}}$, Scalp Hair Assessment $\mathrm{PRO}^{\mathrm{TM}}$, and other ClinRO and PRO measures for AA in Japan. These findings support the use of these clinically meaningful outcome assessments in AA treatment studies involving Japanese patients.

\section{ACKNOWLEDGEMENTS}

The authors would like to thank the clinicians and patients who participated in this study.

Funding. Eli Lilly and Company (Indianapolis, IN, USA) funded DRG Abacus to conduct this study. DRG Abacus (part of Clarivate) is a provider of health economics, outcomes research, and market access services to the pharmaceutical and medical device industry. Eli Lilly and Company (Indianapolis, IN, USA) funded the journal's Rapid Service Fee.

Medical Writing, Editorial, and Other Assistance. The authors would like to acknowledge Global Perspectives and Breeze for their assistance with recruitment and interview conduct in Japan. The authors would like to also acknowledge the contributions of Yves Dutronc (an employee and stockholder of Eli Lilly and Company), Sarah Knight (an employee of DRG Abacus, part of Clarivate), and Kathleen W. Wyrwich (employee and stockholder of Eli Lilly and Company when the study was conducted, and now an employee and stockholder of Pfizer Inc.). Medical writing assistance was provided by Julia Zolotarjova, MSc, MWC, and editorial assistance by Colleen Dumont, BS, of Evidera/
PPD. Support for this assistance was funded by Eli Lilly and Company (Indianapolis, IN, USA).

Authorship. All named authors meet the International Committee of Medical Journal Editors (ICMJE) criteria for authorship for this article, take responsibility for the integrity of the work as a whole, and have given their approval for this version to be published.

Disclosures. Jake Macey, Helen Kitchen, and Natalie V.J. Aldhouse are employees of DRG Abacus, part of Clarivate, a health economic and outcomes research consultancy for pharmaceutical companies. Russel T. Burge, Emily Edson-Heredia, and Jill S. McCollam are employees and stockholders of Eli Lilly and Company. Yoshitaka Isaka and Hitoe TorisuItakura are employees and stockholders of Eli Lilly Japan K.K.

Prior Presentation. The main findings of this study were presented as a poster at the 44th Annual Meeting of the Japanese Society for Investigative Dermatology, Aomori, Japan, November 8-10, 2019.

Compliance with Ethics Guidelines. Study procedures and materials aligned with best practice ethical principles for research involving human participants. The patient interview study protocol was approved by the Western Institutional Review Board in the US (ref: 20182441), which provided the single required ethical review of the study per Japanese guidelines.

Data Availability. The datasets generated and/or analyzed during the current study are not publicly available due to the confidential nature of the information. However, the corresponding author can be contacted for any datarelated questions.

Open Access. This article is licensed under a Creative Commons Attribution-NonCommercial 4.0 International License, which permits any non-commercial use, sharing, adaptation, distribution and reproduction in any medium or format, as long as you give appropriate credit 
to the original author(s) and the source, provide a link to the Creative Commons licence, and indicate if changes were made. The images or other third party material in this article are included in the article's Creative Commons licence, unless indicated otherwise in a credit line to the material. If material is not included in the article's Creative Commons licence and your intended use is not permitted by statutory regulation or exceeds the permitted use, you will need to obtain permission directly from the copyright holder. To view a copy of this licence, visit http://creativecommons.org/licenses/by$\mathrm{nc} / 4.0 /$.

\section{REFERENCES}

1. Simakou T, Butcher JP, Reid S, Henriquez FL. Alopecia areata: a multifactorial autoimmune condition. J Autoimmun. 2019;98:74-85.

2. Lee HH, Gwillim E, Patel KR, et al. Epidemiology of alopecia areata, ophiasis, totalis, and universalis: a systematic review and meta-analysis. J Am Acad Dermatol. 2020;82:675-82.

3. Furue M, Yamazaki S, Jimbow K, et al. Prevalence of dermatological disorders in Japan: a nationwide, cross-sectional, seasonal, multicenter, hospitalbased study. J Dermatol. 2011;38:310-20.

4. Liu LY, King BA, Craiglow BG. Health-related quality of life (HRQOL) among patients with alopecia areata (AA): a systematic review. J Am Acad Dermatol. 2016;75:806-12.e3.

5. Liu LY, King BA, Craiglow BG. Alopecia areata is associated with impaired health-related quality of life: a survey of affected adults and children and their families. J Am Acad Dermatol. 2018;79:556-8. e1.

6. Rencz F, Gulacsi L, Pentek M, Wikonkal N, Baji P, Brodszky V. Alopecia areata and health-related quality of life: a systematic review and meta-analysis. Br J Dermatol. 2016;175:561-71.

7. Abedini R, Hallaji Z, Lajevardi V, Nasimi M, Karimi Khaledi M, Tohidinik HR. Quality of life in mild and severe alopecia areata patients. Int J Womens Dermatol. 2018;4:91-4.

8. Davis DS, Callender VD. Review of quality of life studies in women with alopecia. Int $\mathrm{J}$ Womens Dermatol. 2018;4:18-22.
9. Aldhouse NVJ, Kitchen H, Knight S, et al. "'You lose your hair, what's the big deal?' I was so embarrassed, I was so self-conscious, I was so depressed:" a qualitative interview study to understand the psychosocial burden of alopecia areata. J Patient Rep Outcomes. 2020;4:76.

10. Park H, Yu D-A, Kwon O. Janus kinase inhibitors: an innovative treatment for alopecia areata. J Dermatol. 2019;46:724-30.

11. Tsuboi R, Itami S, Manabe M, et al. Japanese Dermatological Association guidelines for treatment of alopecia areata 2017. Jpn J Dermatol. 2017;127: 2741-62 ([In Japanese]).

12. Hordinsky M, Donati A. Alopecia areata: an evidence-based treatment update. Am J Clin Dermatol. 2014;15:231-46.

13. ClinicalTrials.gov. A study of baricitinib (LY30009104) in adults with severe or very severe alopecia areata (BRAVE-AA2). National Library of Medicine (US); 2019. https://clinicaltrials.gov/ct2/ show/NCT03899259. Accessed 6 Oct 2020.

14. ClinicalTrials.gov. PF-06651600 for the treatment of alopecia areata (ALLEGRO-2b/3). National Library of Medicine (US); 2018. https:// clinicaltrials.gov/ct2/show/NCT03732807. Accessed 6 Oct 2020.

15. ClinicalTrials.gov. A study of baricitinib (LY3009104) in participants with severe or very severe alopecia areata (BRAVE-AA1). National Library of Medicine (US); 2018. https:// clinicaltrials.gov/ct2/show/NCT03570749. Accessed 6 Oct 2020.

16. ClinicalTrials.gov. Long-term PF-06651600 for the treatment of alopecia areata (ALLEGRO-LT). National Library of Medicine (US); 2019. https:// clinicaltrials.gov/ct2/show/NCT04006457. Accessed 6 Oct 2020

17. Xing L, Dai Z, Jabbari A, et al. Alopecia areata is driven by cytotoxic $T$ lymphocytes and is reversed by JAK inhibition. Nat Med. 2014;20:1043-9.

18. Mackay-Wiggan J, Jabbari A, Nguyen N, et al. Oral ruxolitinib induces hair regrowth in patients with moderate-to-severe alopecia areata. JCI Insight. 2016;1:e89790.

19. Craiglow BG, Liu LY, King BA. Tofacitinib for the treatment of alopecia areata and variants in adolescents. J Am Acad Dermatol. 2017;76:29-32.

20. Liu LY, Craiglow BG, Dai F, King BA. Tofacitinib for the treatment of severe alopecia areata and variants: a study of 90 patients. J Am Acad Dermatol. 2017;76:22-8. 
21. Jabbari A, Sansaricq F, Cerise J, et al. An open-label pilot study to evaluate the efficacy of tofacitinib in moderate to severe patch-type alopecia areata, totalis, and universalis. J Investig Dermatol. 2018;138:1539-45.

22. Liu LY, King BA. Tofacitinib for the treatment of severe alopecia areata in adults and adolescents. J Investig Dermatol Symp Proc. 2018;19:S18-20.

23. Food and Drug Administration. Guidance for industry patient-reported outcome measures: use in medical product development to support labeling claims. 2009. https://www.fda.gov/media/77832/ download. Accessed 6 Oct 2020.

24. Food and Drug Administration. Patient-focused drug development: collecting comprehensive and representative input guidance for industry, Food and Drug Administration staff, and other stakeholders. 2018. https://www.fda.gov/media/139088/ download. Accessed 6 Oct 2020.

25. Food and Drug Administration. Patient-focused drug development: methods to identify what is important to patients guidance for industry, Food and Drug Administration staff, and other stakeholders. 2019. https://www.fda.gov/media/131230/ download. Accessed 6 Oct 2020.

26. Wyrwich KW, Kitchen H, Knight S, et al. The Alopecia Areata Investigator's Global Assessment scale: a measure for evaluating clinically meaningful success in clinical trials. $\mathrm{Br} \mathrm{J}$ Dermatol. 2020;183:702-9.

27. Wyrwich KW, Kitchen H, Knight S, et al. Development of the Scalp Hair Assessment PRO ${ }^{\mathrm{TM}}$ measure for alopecia areata. Br J Dermatol. 2020;183(6): 1065-72.
28. Olsen EA, Hordinsky MK, Price VH, et al. Alopecia areata investigational assessment guidelines-part II. National Alopecia Areata Foundation. J Am Acad Dermatol. 2004;51:440-7.

29. Wyrwich KW, Kitchen H, Knight S, et al. Development of clinician-reported outcome (ClinRO) and patient-reported outcome (PRO) measures for eyebrow, eyelash and nail assessment in alopecia areata. Am J Clin Dermatol. 2020;21:725-32.

30. Wyrwich KW, Kitchen H, Knight S, et al. The role of patients in alopecia areata endpoint development: understanding physical signs and symptoms. J Investig Dermatol Symp Proc. 2020;20:S71-7.

31. Patrick DL, Burke LB, Gwaltney CJ, et al. Content validity-establishing and reporting the evidence in newly developed patient-reported outcomes (PRO) instruments for medical product evaluation: ISPOR PRO Good Research Practices Task Force report: part 2-assessing respondent understanding. Value Health. 2011;14:978-88.

32. Willis GB. Cognitive interviewing: a tool for improving questionnaire design. New York: Sage; 2004.

33. Braun V, Clarke V. Using thematic analysis in psychology. Qual Res Psychol. 2006;3:77-101.

34. Guest G, MacQueen KM, Namey EE. Applied thematic analysis. New York: Sage; 2011.

35. Ritchie J, Lewis J, Nicholls CM, Ormston R. Qualitative research practice: a guide for social science students and researchers. New York: Sage; 2013. 